\title{
Dose-Dense Chemotherapy in High-Risk Breast Cancer: Treatment Outcome and Toxicity
}

\author{
Salah El-Mesidy, Mohsen Mokhtar, Amr El-Kashif and Loay Kasem. \\ Department of Clinical Oncology, Faculty of Medicine, Cairo University.
}

\begin{abstract}
Purpose: To prospectively assess the treatment outcome of dose-dense adjuvant doxorubicin and cyclophosphamide (AC) followed by paclitaxel (T) in high-risk breast cancer patients (dose-dense arm) and to compare it with the available treatment results of the conventionally scheduled fluorouracil, doxorubicin and cyclophosphamide (FAC) used to treat high-risk patients (historical control arm). Study endpoints included relapse-free survival (RFS), overall survival (OS) and toxicity.

Patients and Methods: After mastectomy or breast conservative surgery, high-risk node-positive breast cancer patients were assigned to receive adjuvant 4 cycles of doxorubicin/cyclophosphamide followed by 4 cycles of paclitaxel (AC/T) every 2 weeks. The treatment outcome of dose-dense AC/T was compared with that of the conventionally treated high-risk patients using adjuvant 6 cycles of FAC combination chemotherapy scheduled every 3 weeks. The relevant data of patients received FAC chemotherapy were obtained from the medical records. Both treatment groups were balanced regarding age, menopausal status, number of positive axillary lymph nodes and hormonal status.
\end{abstract}

Results: At a median follow up of 37 months (range 12 - 48 months), the 3-year adjusted RFS rates for AC-T and FAC were $76 \%$ and $54.6 \%$, respectively $(\mathrm{P}=0.04)$ and the mean disease-free interval was $40.6 \pm 7.2$ months (95\% CI, 37.7 - 43.6) for dose-dense AC/T arm Vs $36.9 \pm 6.9$ months (95\% CI, 32.3 - 41.5) for FAC arm, $(\mathrm{P}=0.042)$. The subgroup analysis revealed that dose-dense chemotherapy had a statistically significant positive effect on the 3-year RFS in premenopausal patients, patients with 10 or more (N3) positive axillary lymph nodes, positive ER status. There was no statistically significant difference in the 3-year OS between the two regimens. The dose-dense arm was associated with more grade 3-4 toxicity than the control group, mainly neuropathy $(36 \%$. Vs $2.3 \%, \mathrm{P}=0.002)$, anemia $(49 \%$. Vs $13.9 \%, \mathrm{P}=0.037)$ and granulocytopenia $(57 \%$. Vs $213 \%, \mathrm{P}=0.04)$. No toxicity-related mortality was observed in both groups.

Conclusion: Dose-dense AC/T significantly improved the relapse-free survival in patients with high-risk primary breast cancer and was less well tolerated compared with the conventionally scheduled FAC (historical control). The benefit was evident in premenopausal patients, extensive axillary nodal metastasis and positive ER status.

Key words: dose-dense, adjuvant chemotherapy, breast cancer.

Corresponding author: Salah El Mesidy_ E-mail: Salahelmesidy@hotmail.com

\section{INTRODUCTION}

The value of adjuvant chemotherapy has been convincingly demonstrated in breast cancer patients with involved axillary nodes. In the last three decades, incremental benefits in breast cancer disease-free survival (DFS) and overall survival (OS) have occurred with the introduction of anthracycline and taxane adjuvant chemotherapy regimens and with the exploration of higher doses (dose intense) and more frequent administration of these drugs (dose dense) ${ }^{1-3}$. The importance of dose-intensity for adjuvant chemotherapy in patients with breast cancer has first been described by Hryniuk et al. ${ }^{4}$. Higher dose-intensity can be achieved by either increasing the single dose per cycle (i.e. higher dose) or by reducing the intervals between cycles (i.e. dose density) ${ }^{5}$.
The concept called log-cell kill postulated that a given dose of an antineoplastic compound would always kill a certain fraction of the tumor, regardless of the number of cells present. On the basis of that model, higher doses are supposed to be more effective because the fraction of destroyed cancer cells will increase with intensification of the dose. However, the impact of therapy is not only associated with the tumor-cell kill by each dose but also with the rate of cancer regrowth between cycles ${ }^{6}$. Gompertzian kinetics suggest that micrometastases in the adjuvant setting grow faster than established macrometastases; thus, there is likely a higher regrowth of micrometastases between the cycles. Therefore, the administration of cytotoxic drugs with a shortened interval between treatments would be an even more 
effective strategy for minimizing residual tumor burden than pure dose escalation ${ }^{5}$.

In the National Cancer Institute of Canada (NCIC) Clinical Trials Group (CTG) MA5 trial, 6 months of cyclophosphamide, epirubicin and fluorouracil (CEF) improved 5-year DFS and OS compared with 6 months of cyclophosphamide, methotrexate and fluorouracil $(\mathrm{CMF})^{7}$. Subsequently, CEF was compared with a $12-$ week regimen of epirubicin and cyclophosphamide (EC) administered with higher doses and more frequent dosing in locally advanced breast cancer. No difference was detected between regimens in progression-free survival at 34 months $^{8}$. Incorporating taxanes into anthracycline-based schedules yielded, in most studies, an additional benefit in both DFS and OS and dose-dense anthracycline-based and paclitaxel-based regimens have shown to be more effective than the conventional dosing schedule ${ }^{9-13}$.

The aim of the current study was to prospectively assess the treatment outcome of dose-dense adjuvant doxorubicin and cyclophosphamide (AC) followed of paclitaxel (T) in high-risk breast cancer patients. (dose dense arm) Furthermore, the results of dosedense chemotherapy were compared with those of the conventionally scheduled combination chemotherapy of fluorouracil, doxorubicin and cyclophosphamide (FAC) used to treat high-risk patients (control arm). Study endpoints included relapse-free survival (RFS), overall survival (OS) and toxicity.

\section{PATIENTS AND METHODS}

The current prospective study included female patients with histologically proven node-positive highrisk invasive early breast cancer referred to Kasr ElAini Center of Clinical Oncology and Nuclear Medicine (NEMROCK) during the period between January 2006 and December 2009. The study had the institutional ethical review board approval. Files included as historical control were obtained from medical records treated in the same interval.

Patient population: Patients eligible to receive dosedense AC/T chemotherapy had the following criteria:

- $\quad$ Age 18 - 65 years old.

- Performance Status (PS) of 0-2 according to The Eastern Cooperative Oncology Group (ECOG) scale.

- Operable breast cancer: T1-3, N1-2 treated by either mastectomy or breast conservative surgery (BCS) and axillary nodal dissection with free surgical margins.
- High-risk features: either 4 or more positive axillary lymph nodes or 1-3 positive nodes with over expression (score $3+$ ) of the human epidermal growth factor receptor 2 (HER2/neu).

- No evidence of metastatic disease.

- No prior chemotherapy or radiotherapy received.

- Adequate organ functions and No evidence of significant illness or co-morbidities.

- Written informed consent must be obtained.

Pre chemotherapy investigations included;

1. Laboratory investigations: complete blood picture, kidney function tests, liver function tests, CA15-3.

2. Radiological assessment: baseline chest radiograph, abdominal ultrasound, echocardiography and Isotopic bone scanning.

3. Estrogen and progesterone receptors (ER and PR) status.

4. HER2/neu expression by immunohistochemistry: a score of $(3+)$ was considered positive (overexpressed) while scores of $(0,1+$, or $2+)$ were considered negative.

Treatment regimen: Eligible patients were assigned to receive dose-dense $\mathrm{AC} / \mathrm{T}$ adjuvant chemotherapy which consisted of: 4 cycles of AC (doxorubicin $60 \mathrm{mg}$ / $\mathrm{m} 2$ and cyclophosphamide $600 \mathrm{mg} / \mathrm{m} 2$ administered IV on day 1) every 2 weeks followed by 4 cycles of T (paclitaxel $175 \mathrm{mg} / \mathrm{m} 2$ administered by IV infusion over 3 hours on day 1) every 2 weeks. Prophylactic granulocyte colony-stimulating factor (G-CSF) was administered using filgrastim (5 ug/Kg/day subcutaneous injection) for 3 consecutive days 24-48 hours after chemotherapy. Treatment-induced toxicity was evaluated according to the National Cancer Institute (NCI) Common Toxicity Criteria version 2.0 grading system ${ }^{14}$. Patients who had an overexpressed HER2/neu did not receive adjuvant trastuzumab.

After completion of chemotherapy, radiotherapy was given to the whole breast (after BCS) or chest wall ( after mastectomy) and peripheral lymphatics at a dose of 5040 cGy/28 fractions using linear accelerator $6 \mathrm{MV}$ followed by a boost to the site of primary tumor(after BCS) at a dose of 1600-2000 cGy/8-10 fractions by electron beam or photons. Premenopausal patients with ER and/or PR positive tumors were assigned to receive 5 years of adjuvant tamoxifen $20 \mathrm{mg}$ /day while postmenopausal women with positive receptors were assigned to receive 5 years of sequential aromatase inhibitor (AI) and tamoxifen.

Follow-Up: After completion of chemotherapy and radiotherapy, patients were planned for follow up visits every 3 months during the first year, every 4 months 
in the second year and every 6 months until the end of the fifth year. Patients were subjected to careful historytaking and physical examination. A mammogram and echocardiography were performed yearly whereas, chest $\mathrm{X}$-ray, abdominal ultrasound or bone scan were requested if symptoms or laboratory abnormalities were present.

Outcome Measures: The primary end point of this study was relapse-free survival (RFS) which was defined as the time from surgery to documented recurrence. Local breast recurrence, nodal recurrence and metastatic disease were considered as a recurrence. Patients who had a new contralateral primary breast cancer or a second malignancy were not considered to have a relapse. Secondary outcome measures included overall survival (OS) which was defined as the time from surgery to documented death due to any cause. Treatment-related toxicity is another secondary end point.

AC/T versus FAC analysis: The treatment outcome of dose-dense AC/T was eventually compared with the available treatment results of the conventionally treated high-risk node-positive patients using adjuvant 6 cycles of FAC combination chemotherapy (5-flurouracil 600mg/ $\mathrm{m} 2$, doxorubicin $60 \mathrm{mg} / \mathrm{m} 2$ and cyclophosphamide 600 $\mathrm{mg} / \mathrm{m} 2$ were administered IV on day 1) scheduled every 3 weeks. Patients received FAC chemotherapy enrolled in the analysis had the same inclusion criteria and were treated during a defined period of time with a comparable follow-up period to assess the same outcome measures. The relevant data including the clinico-pathological characteristics, outcome parameters and treatmentrelated toxicity were obtained from the medical records.

Statistical analysis: Data were statistically described in terms of range, mean \pm standard deviation $( \pm \mathrm{SD})$, frequencies (number of cases) and percentages when appropriate. Survival analysis was done for the different outcome measures using Kaplan Maier statistics calculating the mean and median survival time for each group with their $95 \% \mathrm{CI}$ and the corresponding survival graphs. Factors examined as a stepwise adjusted Kaplan Maier were age at presentation $(\leq 35,35-49, \geq 50$ years), T status (T1, T2, T3, T4), N status (N1, N2, N3), menopausal status (postmenopausal or premenopausal), ER status (negative or positive), PR status (negative or positive) and HER2/neu status (negative or amplified). A plot of RFS and OS Vs the mentioned factors were calculated and compared between the two groups. A probability value ( $\mathrm{p}$ value) less than 0.05 was considered statistically significant. All statistical calculations were done using computer programs Microsoft Excel 2007 (Microsoft Corporation, NY, USA) and SPSS (Statistical Package for the Social Science; SPSS Inc., Chicago, IL, USA) version 17 for Microsoft Windows.

\section{RESULTS}

A total of 61 patients were recruited to receive dose dense AC-T and were compared with files of 43 patients received standard FAC chemotherapy with respect to the clinico-epidemiological features, disease and treatmentrelated factors. The follow-up period ranged from 12 months to 48 months with a median follow up of 37 months. Baseline characteristics were balanced between both treatment arms with no statistically significant differences Table (1).

Fifty out of the 61 patients $(81.9 \%)$ in the dose dense arm completed the 8 cycles of chemotherapy within 110 days (planned time between the first and the last cycles). The delay in the majority of patients was mainly due to treatment-related toxicity (neuropathy, cytopenia and febrile neutropenia) and rarely patient non-compliance. Dose reduction due to grade 3-4 toxicity was reported in 8 patients $(13.1 \%)$. On the other hand, 39 out of the 43 patients $(90.6 \%)$ in the FAC arm completed the 6 cycles within 110 days and most of the treatment delay was due to febrile neutropenia. Dose reduction due to hematological toxicity was required only in one patient $(2.8 \%)$. The mean chemotherapy duration was 123 days $(\mathrm{SD} \pm 5.9)$ in the dose dense $\mathrm{AC} / \mathrm{T}$ arm compared to 117 days $(\mathrm{SD} \pm 6.3$ ) in the control FAC arm (borderline significance $\mathrm{P}=0.05$ ).

The 3-year adjusted RFS rates for AC-T and FAC were $76 \%$ and $54.6 \%$, respectively $(\mathrm{P}=0.04)$ Figure (1). The mean disease-free interval was 40.6 months $(\mathrm{SD} \pm 7.2)$ (95\% CI, 37.7 - 43.6) for dose-dense AC/T arm Vs 36.9 months (SD \pm 6.9$)(95 \% \mathrm{CI}, 32.3$ - 41.5) for FAC arm, $(\mathrm{P}=0.040)$. Regarding the pattern of relapse, systemic relapse (metastatic disease) was the predominant pattern in both arms (87.8\% for dose-dense AC/T arm and 78.6\% for FAC arm; $\mathrm{p}=0.041$ ).

The subgroup analysis revealed that dose-dense chemotherapy had a statistically significant positive effect on 3-year RFS in premenopausal patients $(77.5 \%$ for $\mathrm{AC} / \mathrm{T}$ arm Vs $54 \%$ for $\mathrm{FAC}$ arm, $\mathrm{P}=0.045$ ), patients with 10 or more (N3) positive axillary lymph nodes (72\% for AC/T arm Vs $26 \%$ for FAC arm, $\mathrm{P}=0.02$ ) and positive ER status $(83 \%$ for AC/T arm Vs $50 \%$ for FAC arm, $\mathrm{P}=0.017)$. HER $2 /$ neu status did not significantly affect the 3-year RFS in patients received dose-dense $\mathrm{AC} / \mathrm{T}$ though there was a trend towards better outcome compared with FAC arm in HER2/neu positive patients (77\% for AC/T arm Vs 57\% for FAC arm, $\mathrm{P}=0.051$ ).

There was no statistically significant difference in the 3-year OS between the two regimens or subgroups Figure (2). Mean OS was $46.1 \pm 5.6$ months for FAC arm compared to $43 \pm 6.0$ months in the dose dense arm 
$(\mathrm{P}=0.7)$. Two patients in the FAC arm and 3 patients in the $\mathrm{AC} / \mathrm{T}$ arm died due to extensive metastatic disease (mainly pulmonary and liver metastases).

Hematologic toxicity was more pronounced in the dose dense arm. The difference was highly significant $(\mathrm{P}=0.01)$ with respect to anemia, granulocytopenia and thrombocytopenia. In the $\mathrm{AC} / \mathrm{T}$ arm, the incidence of hematological toxicity was more evident during treatment with paclitaxel rather than AC. All patients had full recovery of their peripheral-blood counts at the end of treatment. No toxicity-related mortality was observed in both arms. Non hematologic toxicity occurred significantly more often in the dose dense arm. For example, peripheral sensory neuropathy grade 3-4 was more evident in patients received $\mathrm{AC} / \mathrm{T} 36 \% \mathrm{Vs} 2.3 \%$ in FAC arm $(\mathrm{P}=0.002)$ (Table 2$)$. After a median followup period of more than 3 years, only $2 \%$ of the patients received dose-dense chemotherapy had persistent grade 1 or 2 neurotoxicity compared with none in the FAC arm. In both arms, none of the patients developed clinically evident congestive heart failure or significant reduction in the ejection fraction. Furthermore, there were no recorded cases of secondary malignancy, leukemia or myelodysplastic syndrome (MDS) during the short follow up period.

Table 1: Baseline patient and tumor characteristics.

\begin{tabular}{|c|c|c|c|c|c|}
\hline & \multicolumn{2}{|c|}{ FAC $($ no. $=43)$} & \multicolumn{2}{|c|}{$\mathrm{AC}-\mathrm{T}(\mathrm{no} .=61)$} & \multirow[t]{2}{*}{ P value } \\
\hline & No. & $\%$ & No. & $\%$ & \\
\hline \multicolumn{6}{|l|}{ Age (years) } \\
\hline$\leq 35$ & 3 & 7 & 8 & 13.1 & \\
\hline $36-50$ & 21 & 48.8 & 25 & 41 & \\
\hline $50+$ & 19 & 44.2 & 28 & 45.9 & 0.53 \\
\hline Median (range) & \multicolumn{2}{|c|}{$47.5(27-65)$} & \multicolumn{2}{|c|}{$47(24-63)$} & 0.6 \\
\hline \multicolumn{6}{|c|}{ Menopausal status } \\
\hline Pre menop. & 20 & 46.5 & 38 & 62.3 & \\
\hline Post menop. & 23 & 53.5 & 23 & 37.7 & 0.81 \\
\hline \multicolumn{6}{|l|}{ Grade } \\
\hline G1 & 2 & 4.65 & 2 & 3.3 & \\
\hline G2 & 39 & 90.70 & 54 & 91.5 & \\
\hline G3 & 2 & 4.65 & 5 & 8.2 & 0.2 \\
\hline \multicolumn{6}{|l|}{ Type of surgery } \\
\hline MRM & 32 & 74.5 & 54 & 88.5 & \\
\hline $\mathrm{BCS}$ & 11 & 25.5 & 7 & 11.5 & 0.16 \\
\hline \multicolumn{6}{|l|}{ T-stage } \\
\hline $\mathrm{T} 1$ & 5 & 11.6 & 8 & 13.1 & \\
\hline $\mathrm{T} 2$ & 26 & 60.4 & 39 & 63.9 & \\
\hline $\mathrm{T} 3$ & 5 & 11.6 & 10 & 16.4 & \\
\hline $\mathrm{T} 4$ & 7 & 16.4 & 4 & 6.6 & 0.41 \\
\hline \multicolumn{6}{|l|}{ N-stage } \\
\hline N1 & 3 & 7 & 6 & 9.8 & \\
\hline $\mathrm{N} 2$ & 23 & 53.5 & 33 & 54.1 & \\
\hline N3 & 17 & 39.5 & 22 & 36.1 & 0.85 \\
\hline \multicolumn{6}{|l|}{ Receptor status } \\
\hline $\mathrm{ER}+\mathrm{ve}$ & 35 & 81.4 & 44 & 72.1 & \\
\hline ER -ve & 8 & 18.6 & 17 & 27.9 & 0.19 \\
\hline$P R+v e$ & 33 & 76.7 & 41 & 67.2 & \\
\hline PR -ve & 10 & 23.3 & 20 & 32.8 & 0.22 \\
\hline \multicolumn{6}{|l|}{ HER2/neu } \\
\hline Negative & 38 & 88.4 & 51 & 83.6 & \\
\hline Positive & 5 & 11.6 & 10 & 16.4 & 0.76 \\
\hline
\end{tabular}


Table 2: Treatment-related toxicity (G III/IV) in both groups.

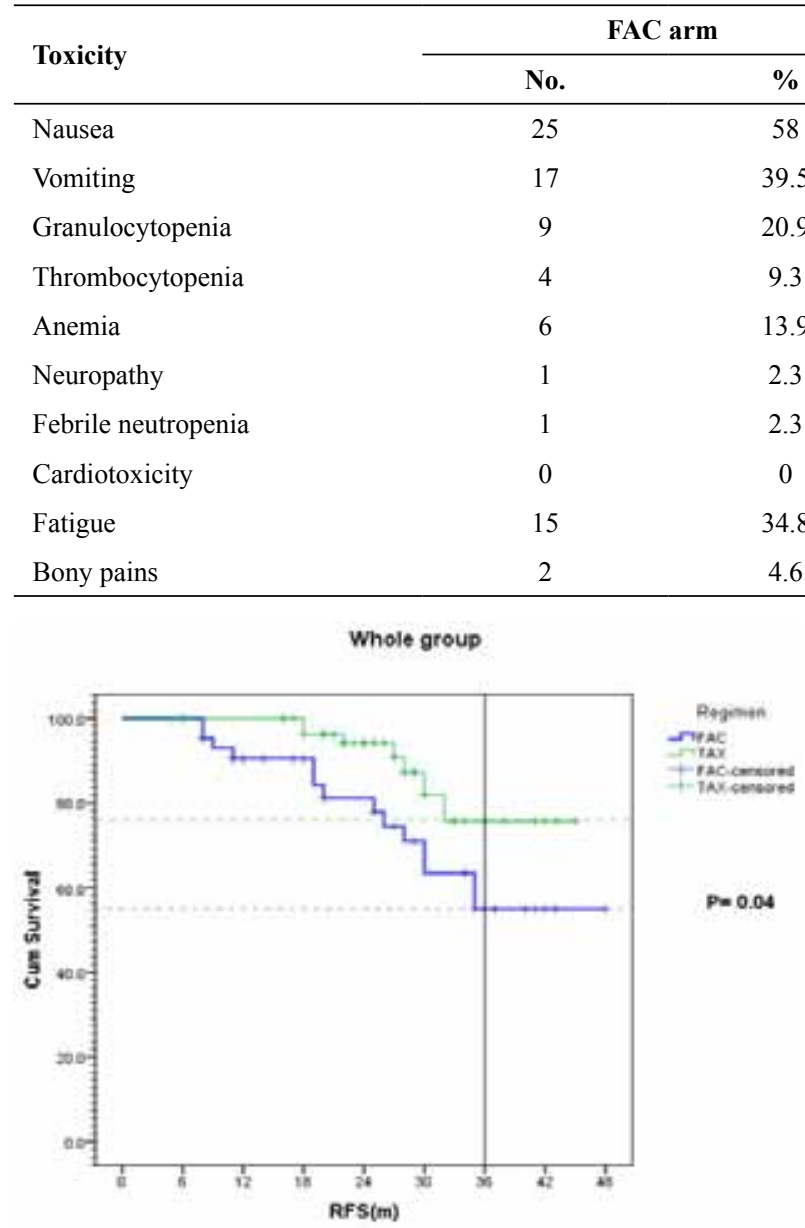

Figure 1: Kaplan-Maier survival curve of relapse-free survival in both treatment groups.

\section{DISCUSSION}

The outcomes of women with early breast cancer have improved incrementally through trials that have evaluated new chemotherapy drugs and different doses and schedules of drugs postoperatively. Although much progress has been made, women still develop recurrences ${ }^{15}$. Dose-dense chemotherapy has become one of the possible standards of adjuvant chemotherapy ${ }^{16}$. The hypothesis that such a strategy is effective was based, in part, on theories developed by Skipper and by Norton and Simon ${ }^{17-19}$. In their experimental models, a given dose of drug always kills a certain fraction, rather than a certain number, of exponentially growing cancer cells. However, breast cancer cells proliferate by nonexponential gompertzian kinetics and the rate of cancer cell proliferation between treatment cycles is more rapid than that used in exponential models ${ }^{19}$.

The current study was designed to assess the treatment outcome of adjuvant dose-dense $\mathrm{AC} / \mathrm{T}$ in high-

\begin{tabular}{lccc} 
& & AC-T arm & P value \\
\hline 8 & No. & \% & \\
\hline 5 & 50 & 81 & 0.03 \\
.9 & 36 & 59 & 0.04 \\
3 & 35 & 57 & 0.031 \\
.9 & 3 & 5 & 0.4 \\
3 & 30 & 49 & 0.032 \\
3 & 22 & 36 & 0.02 \\
0 & 3 & 5 & 0.08 \\
.8 & 0 & 0 & 0.1 \\
6 & 15 & 24.5 & 0.8
\end{tabular}

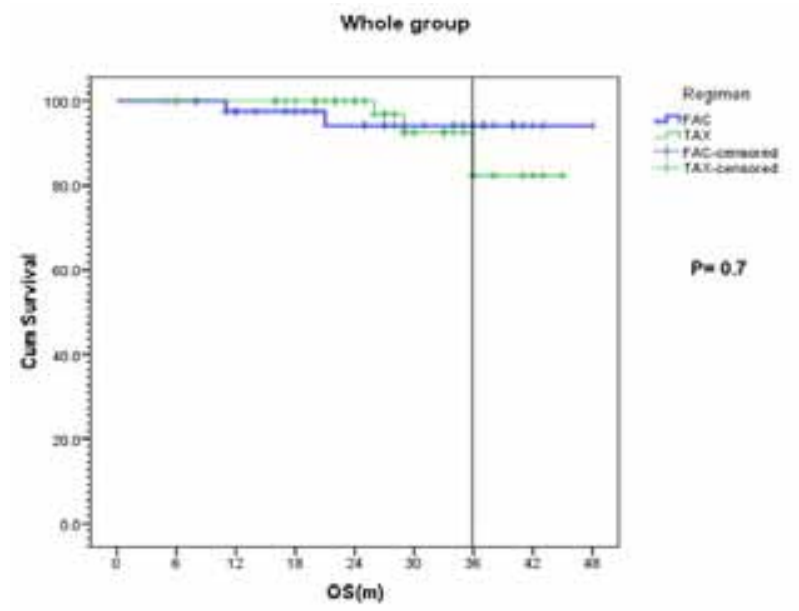

Figure 2: Kaplan-Maier survival curve of overall survival in both treatment groups

risk breast cancer patients and to identify the possible subgroup of patients that had a significant improvement of their survival parameters. Due to the growing evidence from recent clinical trials that showed improvement of treatment outcome in favor of dose-dense compared to conventionally scheduled chemotherapy, prospective data of $\mathrm{AC} / \mathrm{T}$ were compared with the available data of the classic FAC regimen used to be the standard adjuvant treatment for high-risk patients. Baseline characteristics were balanced between both treatment arms with no statistically significant differences.

The results of the current study revealed that dosedense AC/T was superior to conventional FAC in terms of RFS. The benefit was evident in premenopausal patients, patients with 10 or more $(\mathrm{N} 3)$ positive axillary lymph nodes and positive ER status, whereas the benefit was not affected by the HER2/neu status. There was no statistically significant difference in the 3-year OS between the two regimens. Several clinical trials have confirmed the importance of dose density and dose 
intensity in the adjuvant treatment of early breast cancer, especially in women with positive nodal status.

The Cancer and Leukemia Group B (CALGB) trial C9741 compared 4 cycles of AC followed by 4 cycles of paclitaxel in dose-dense intervals with conventional 3 -week intervals in 1,973 node-positive patients and found that the dose-dense regimen produced significantly better DFS ( $\mathrm{HR}=0.74 ; \mathrm{P}=.010)$ and $\mathrm{OS}(\mathrm{HR}=0.69$; $\mathrm{p}=.013)$. Severe neutropenia was observed less frequently in patients receiving G-CSF support with the dose-dense chemotherapy schedule and the regimen was otherwise well tolerated ${ }^{12}$. This finding was recently challenged by an extensive retrospective analysis of this and two other CALGB trials, in which patients who were estrogen or progesterone receptor positive and received tamoxifen after chemotherapy experienced a markedly smaller effect of dose-dense delivery of chemotherapy, with a minimal reduction in the relative risk for recurrence and no reduction in the risk for death ${ }^{20}$.

The German Arbeitsgemeinschaft Gastrointestinal Onkologie (AGO) trial examined 1,284 patients with more than four positive lymph nodes using treatment with a dose-dense, dose-intense regimen of epirubicin, paclitaxel and cyclophosphamide (E-T-C) administered sequentially on a 2-week schedule with GCSF support. These very high risk patients achieved 2- year RFS rates of $85 \%$, versus $82 \%$ for conventionally dosed EC $+\mathrm{T}(\mathrm{p}=.046$, two-tailed test). Survival benefits were observed irrespective of hormone receptor status or HER-2 expression, but were particularly impressive in women with 10 or more positive lymph nodes. That trial used epirubicin doses of $150 \mathrm{mg} / \mathrm{m} 2$ every 2 weeks for a cumulative epirubicin dose of $450 \mathrm{mg} / \mathrm{m} 2$ in the dosedense $\operatorname{arm}^{21}$.

Many studies have suggested that the benefit of taxane-based or dose-dense chemotherapy may be restricted to patients with HER2-positive or steroid hormone receptor- negative disease ${ }^{22,23}$. The current study failed to demonstrate such benefit as the dose-dense regimen had a positive effect in patients with estrogen receptor-positive breast cancer with no effect of HER2/ neu status. The possible explanation could be the higher frequency of $\mathrm{N} 3$ disease (36\%) among patients received $\mathrm{AC} / \mathrm{T}$, which may have minimized the effect of biological predictors of response (HER2/neu status) and maximized the effect of clinico-pathological factors (nodal status).

However, not all adjuvant dose-dense trial data were viewed as positive. The GONO-MIG (Gruppo Oncologico Nord Ovest- Mammella InterGruppo) trial compared FU, epirubicin and cyclophosphamide administered every 3 weeks versus the same regimen given every 2 weeks with granulocyte colony-stimulating factor support. As in the CALGB study, the chemotherapy dose per cycle and total number of cycles were held constant and only the interval between cycles varied. The difference between these two arms did not reach statistical significance for either recurrence or death ${ }^{24}$. These negative results may be explained by the study design. The GONO-MIG trial applied a suboptimal version of the FEC regimen (ie, FU at $600 \mathrm{mg} / \mathrm{m} 2$, epirubicin at $60 \mathrm{mg} / \mathrm{m} 2$ and cyclophosphamide at $600 \mathrm{mg} / \mathrm{m} 2$ six cycles). The total dose of epirubicin in both arms was $360 \mathrm{mg} / \mathrm{m} 2$, which corresponded to only $50 \%$ of the total dose in the highly effective Canadian CEF regimen (ie, cyclophosphamide $75 \mathrm{mg} / \mathrm{m} 2$ orally days 1 through 14 , epirubicin $60 \mathrm{mg} / \mathrm{m} 2$ days 1 and 8 and FU 500mg/m2 intravenously day 1and 8 for six cycles) or to only $60 \%$ of the total dose in the French FE100C regimen (FU500mg/m2, epirubicin $100 \mathrm{mg} / \mathrm{m} 2$ and cyclophosphamide $500 \mathrm{mg} / \mathrm{m} 2$ for six cycles $)^{25,26}$.

Hematologic and non hematologic toxicities were more pronounced in the dose-dense AC/T. However, all patients had full recovery of their peripheral-blood counts at the end of treatment and only $2 \%$ of the patients had persistent grade 1 or 2 neurotoxicity. At a median follow up of 37 months, there were no recorded cases of secondary malignancy, leukemia or MDS and this finding does not correspond to published data of anthracycline and cyclophosphamide adjuvant regimens. In the randomized, phase III trial, conducted by Moebus et al, 1,284 patients with four or more involved axillary lymph nodes were randomly assigned to receive intense dose-dense (IDD) sequential epirubicin, paclitaxel and cyclophosphamide (IDD-ETC) every 2 weeks or conventionally scheduled epirubicin/cyclophosphamide followed by paclitaxel every three weeks. Four occurrences $(0.6 \%$ of patients) of secondary leukemia/ MDS were reported with the 5-year follow-up data in the IDD-ETC arm only ${ }^{16}$.

With 10 years of follow-up, 5 occurrences $(1.4 \%$ of patients) of secondary leukemia/MDS were reported for patients receiving the Canadian CEF regimen ${ }^{25}$. Praga et al, reviewed 19 adjuvant trials with epirubicin and cyclophosphamide in 2005. Depending on the total dose of both epirubicin and cyclophosphamide, patients had a 8-year cumulative probability of secondary leukemia/ MDS ranging between $0.37 \%$ and $4.97 \%{ }^{27}$. The absence of secondary leukemia/MDS in the current study may be explained by the relatively short follow- up interval and low total anthracycline dose $(240 \mathrm{mg} / \mathrm{m} 2$ in the dose dense arm and $360 \mathrm{mg} / \mathrm{m} 2$ in the conventional arm).

In conclusion, dose dense $\mathrm{AC} / \mathrm{T}$ is an effective, feasible and safe regimen with manageable toxicity for adjuvant treatment of node-positive high-risk breast 
cancer patients. Regarding efficacy, however, the number of patients and the short follow up interval are not enough to provide the statistical power to the results. Future research should focus on predictive factors for different chemotherapeutic regimens and on combining targeted therapies with dose-dense regimens to continue the incremental advance in the outcomes of women with early breast cancer.

\section{CONCLUSION}

Dose-dense AC/T significantly improved the relapsefree survival in patients with high-risk primary breast cancer and was less well tolerated compared with the conventionally scheduled FAC. The benefit was more evident in premenopausal patients, extensive axillary nodal metastasis and positive ER status.

\section{REFERENCES}

1. Levine $\mathrm{MN}$, Whelan T. Adjuvant chemotherapy for breast cancer--30 years later. N.Engl.J.Med. 2006 Nov 2;355(18):1920-2.

2. Levine M, Eisen A. Anthracycline adjuvant chemotherapy: How much is enough? J.Clin.Oncol. 2001 Feb 1;19(3):599-601.

3. Dang C, Hudis C. Adjuvant taxanes in the treatment of breast cancer: No longer at the tip of the iceberg. Clin. Breast Cancer. 2006 Apr;7(1):51-8.

4. Hryniuk W, Levine MN. Analysis of dose intensity for adjuvant chemotherapy trials in stage II breast cancer. J.Clin.Oncol. 1986 Aug;4(8):1162-70.

5. Norton L. A Gompertzian model of human breast cancer growth. Cancer Res. 1988 Dec 15;48(24 Pt 1):7067-71.

6. Skipper HE. Kinetics of mammary tumor cell growth and implications for therapy. Cancer 1971 Dec;28(6):1479-99.

7. Levine MN, Bramwell VH, Pritchard KI, Norris BD, Shepherd LE, Abu Zahra H, et al. Randomized trial of intensive cyclophosphamide, epirubicin and fluorouracil chemotherapy compared with cyclophosphamide, methotrexate and fluorouracil in premenopausal women with node-positive breast cancer. National Cancer Institute of Canada Clinical Trials Group. J.Clin.Oncol. 1998 Aug;16(8):2651-8.

8. Therasse P, Mauriac L, Welnicka Jaskiewicz M, Bruning $\mathrm{P}, \mathrm{Cufer} \mathrm{T}$, Bonnefoi $\mathrm{H}$, et al. Final results of a randomized phase III trial comparing cyclophosphamide, epirubicin and fluorouracil with a dose-intensified epirubicin and cyclophosphamide + filgrastim as neoadjuvant treatment in locally advanced breast cancer: An EORTC-NCIC-SAKK multicenter study. J.Clin.Oncol. 2003 Mar 1;21(5):843-50.

9. Roche H, Fumoleau P, Spielmann M, Canon JL, Delozier $\mathrm{T}$, Serin D, et al. Sequential adjuvant epirubicin-based and docetaxel chemotherapy for node-positive breast cancer patients: The FNCLCC PACS 01 Trial. J.Clin.Oncol. 2006 Dec 20;24(36):5664-71.
10. Francis $\mathrm{P}$, Crown $\mathrm{J}$, Di Leo A, Buyse M, Balil A, Andersson $\mathrm{M}$, et al. Adjuvant chemotherapy with sequential or concurrent anthracycline and docetaxel: Breast International Group 02-98 randomized trial. J.Natl. Cancer Inst. 2008;100(2):121-33.

11. Mamounas EP, Bryant J, Lembersky B, Fehrenbacher L, Sedlacek SM, Fisher B, et al. Paclitaxel after doxorubicin plus cyclophosphamide as adjuvant chemotherapy for node-positive breast cancer: Results from NSABP B-28. J.Clin.Oncol. 2005 Jun 1;23(16):3686-96.

12. Citron ML, Berry DA, Cirrincione C, Hudis C, Winer EP, Gradishar WJ, et al. Randomized trial of dose-dense versus conventionally scheduled and sequential versus concurrent combination chemotherapy as postoperative adjuvant treatment of node-positive primary breast cancer: first report of Intergroup Trial C9741/Cancer and Leukemia Group B Trial 9741. J.Clin.Oncol. 2003 Apr 15;21(8):1431-9.

13. Hudis C, Citron ML, Berry D, Cirrincione C, Gradishar W, Davidson N, et al. Five year follow-up of INT C9741: Dose Dense (DD) chemotherapy (CRx) is safe and effective. Breast Cancer Res. 2005;94(Suppl. 1):S20.

14. National Cancer Institute. Common toxicity criteria manual. 1999; Available at: http://ctep.cancer.gov/ protocolDevelopment/electronic_applications/docs/ ctcmanual_v4_10-4-99.pdf.

15. Burnell $\bar{M}$, Levine MN, Chapman JAW, Bramwell V, Gelmon K, Walley B, et al. Cyclophosphamide, epirubicin and fluorouracil versus dose-dense epirubicin and cyclophosphamide followed by paclitaxel versus doxorubicin and cyclophosphamide followed by paclitaxel in node-positive or high-risk node-negative breast cancer. J.Clin.Oncol. 2010;28(1):77-82.

16. Moebus V, Jackisch C, Lueck HJ, Du Bois A, Thomssen $\mathrm{C}$, Kurbacher $\mathrm{C}$, et al. Intense dose-dense sequential chemotherapy with epirubicin, paclitaxel and cyclophosphamide compared with conventionally scheduled chemotherapy in high-risk primary breast cancer: Mature results of an AGO phase III study. J.Clin. Oncol. 2010;28(17):2874-88.

17. Skipper HE. Laboratory models: Some historical perspective. Cancer Treat.Rep. 1986 Jan;70(1):3-7.

18. Norton L, Simon R, Brereton HD, Bogden AE. Predicting the course of Gompertzian growth. Nature 1976 Dec 9;264(5586):542-5.

19. Norton L. Theoretical concepts and the emerging role of taxanes in adjuvant therapy. Oncologist 2001;6 Suppl 3:30-5.

20. Berry DA, Cirrincione C, Henderson IC, Citron ML, Budman DR, Goldstein LJ, et al. Effects of improvements in chemotherapy on disease-free and overall survival of estrogen-receptor negative, node-positive breast cancer: 20-year experience of the CALGB \& U.S. Breast Intergroup. Breast Cancer Res Treat 2004;88(Suppl. 1):S69. 
21. Möbus VJ, Untch M, Du Bois A, Lueck HJ, Thomssen C, Kuhn W, et al. Dose-dense sequential chemotherapy with epirubicin(E), paclitaxel (T) and cyclophosphamide (C) (ETC) is superior to conventional dosed chemotherapy in high-risk breast cancer patients $(\geq 4+L N)$. First results of an AGO-trial. Proc.Am.Soc.Clin.Oncol. 2004;22(14S):513.

22. Hayes DF, Thor AD, Dressler LG, Weaver D, Edgerton S, Cowan D, et al. HER2 and response to paclitaxel in nodepositive breast cancer. N.Engl.J.Med. 2007;357(15):1496506.

23. Berry DA, Cirrincione C, Henderson IC, Citron ML, Budman DR, Goldstein LJ, et al. Estrogen-receptor status and outcomes of modern chemotherapy for patients with node-positive breast cancer. JAMA 2006 Apr 12;295(14):1658-67.

24. Venturini M, Del Mastro L, Aitini E, Baldini E, Caroti C, Contu A, et al. Dose-dense adjuvant chemotherapy in early breast cancer patients: results from a randomized trial. J.Natl.Cancer Inst. 2005 Dec 7;97(23):1724-33.
25. Levine MN, Pritchard KI, Bramwell VH, Shepherd LE, Tu D, Paul N. Randomized trial comparing cyclophosphamide, epirubicin and fluorouracil with cyclophosphamide, methotrexate and fluorouracil in premenopausal women with node-positive breast cancer: Update of National Cancer Institute of Canada Clinical Trials Group Trial MA5. J.Clin.Oncol. 2005 Aug 1;23(22):5166-70.

26. Bonneterre J, Roche H, Kerbrat P, Bremond A, Fumoleau $\mathrm{P}$, Namer $\mathrm{M}$, et al. Epirubicin increases long-term survival in adjuvant chemotherapy of patients with poor-prognosis, node-positive, early breast cancer: 10-year follow-up results of the French Adjuvant Study Group 05 randomized trial. J.Clin.Oncol. 2005 Apr 20;23(12):2686-93.

27. Praga C, Bergh J, Bliss J, Bonneterre J, Cesana B, Coombes RC, et al. Risk of acute myeloid leukemia and myelodysplastic syndrome in trials of adjuvant epirubicin for early breast cancer: Correlation with doses of epirubicin and cyclophosphamide. J.Clin.Oncol. 2005 Jun 20;23(18):4179-91. 